\title{
Editorial
}

\section{The Fontan procedure: lessons from the past}

The Fontan circulation was one of the "big ideas" in congenital heart disease in the 1970s. This completely novel ment but, gradually, understanding of its properties progressed and there is now an expanding cohort of patients followed up with stable Fontan circulations.

In 1990, on the basis of an observational study of the survival and wellbeing of 334 patients, Fontan et al came to the disappointing conclusion that even after a perfect Fontan procedure there was a gradual decline in the functional status of patients that the authors could not attribute to anything other than the Fontan circulation itself. ${ }^{1}$ This and other long term follow up studies have provoked a wave of negativity about Fontan-type operations that has had repercussions for patients with complex anatomy facing radical operations.

Two European papers, ${ }^{2}{ }^{3}$ describe cross sectional studies of medium and long term survivors of Fontan-type operations. They both focus on the non-cardiac complications of liver dysfunction, coagulation disorders, and protein losing enteropathy rather than the time related incidences of death, arrhythmias, and surgical complications, such as pathway obstruction, addressed in several other series. We have now to respond to the consensus that at least $40 \%$ of patients, most of whom are functioning tolerably well at late follow up, have a raised concentration of one or more liver enzymes, at least $25 \%$ have a depletion of some coagulation factors, and $7 \%$ have abnormally low serum albumin.

\section{Venous hypertension, liver dysfunction, and low albumin concentrations}

Are the abnormalities of liver function and coagulation explicable by the Fontan state as we understand it? Fundamental to the operation of all Fontan-type circulations is a chronic increase of systemic venous pressure to levels that would correspond to significant right heart disease in a biventricular circulation (fig 1). The association among longstanding systemic venous hypertension, liver dysfunction, and protein losing enteropathy was familiar to cardiologists long before the Fontan operation was conceived. Chronic conditions producing a high enough venous pressure to cause insidious liver and gastrointestinal damage include constrictive pericarditis ${ }^{45}$ and tricuspid valve type of circulation was at first the source of some astonish-

disease. ${ }^{6}$ The full blown clinical picture is dominated by ascites and oedema associated with hypoalbuminaemia but with a background of intestinal lymphatic dysfunction, which is usually clinically silent.

If this cluster of features represents an inevitable consequence of longstanding right atrial hypertension, it is hard to guess where our Fontan patients are along the course of its natural history. Fontan patients will be the first cohort with significant right atrial hypertension that can be followed from a clear starting date, from which actuarial data can be derived. Feldt et al estimate that $13.4 \%$ of 30 day survivors of a Fontan procedure will have biochemical evidence of protein losing enteropathy at 10 years.

ROLE OF THE GUT

The impression from clinical studies of constrictive pericarditis appearing in the $1960 \mathrm{~s}^{4-6}$ is that, like Fontan patients, there was often evidence of mild derangement of synthetic or excretory liver function, but that important symptoms emerged only as the plasma albumin drifted down. Where hypoalbuminaemia and chronic venous hypertension coexisted, the well documented cause was gastrointestinal protein loss. ${ }^{4-6}$ Painstaking studies showed that $3-5 \%$ of the intravenous albumin pool might leak into the gastrointestinal tract daily (normal $0.1-1.7 \%$ ). The patients' livers managed to maintain synthesis at the upper limit of normal, ruling out major liver impairment or malnutrition as the primary cause of hypoalbuminaemia. ${ }^{6}$

\section{LYMPHATIC PHYSIOLOGY}

The enormous capacity of the lymphatic system to shift transuded fluid and the role of the lymphatics in protein turnover has also been extensively investigated. Besides providing channels allowing fluid to move passively downstream along a pressure gradient, and the potential to direct fluid progradely by the provision of valves, the walls of the larger lymphatics are muscularised. The larger lymphatic vessels can pump against a pressure gradient (the acutely compressed human thoracic duct can generate a pulsatile pressure of $30 \mathrm{~mm} \mathrm{Hg}) .^{8}$ The lymphatic pumps appear to have Starling properties, with an ability to increase their pumping rate and stroke volume as they are distended by increasing preload ${ }^{9}$ or afterload. ${ }^{10}$ Like other pumps, this Starling mechanism reaches a physiological

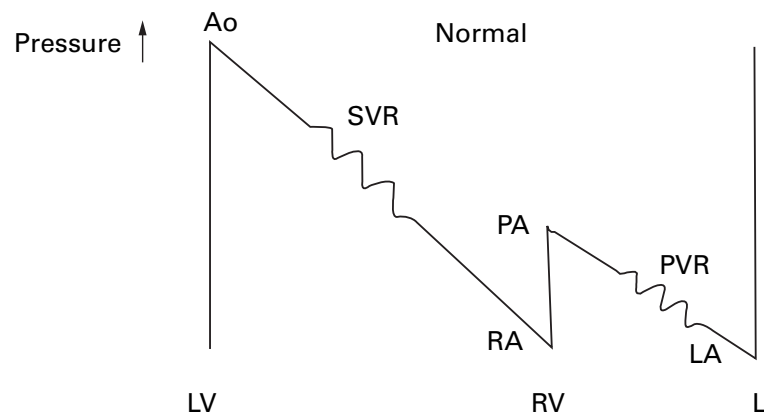

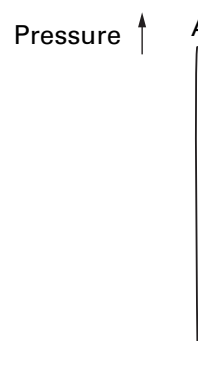

LV

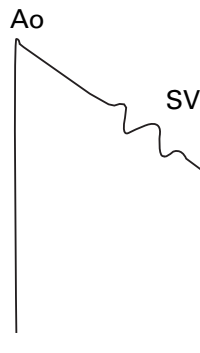

$\mathrm{V}$
Fontan

Figure 1 Pressure relations in normal and Fontan circulations. Ao, aorta; LV, left ventricle; PA, pulmonary artery; PVR, pulmonary vascular resistance; $R A$, right atrium; $R V$, right ventricle; $S V R$, systemic vascular resistance. 
limit of distension (transmural pressure) such that at an outflow pressure of about $16 \mathrm{~mm} \mathrm{Hg}$ pump function ceases completely and only conduit flow occurs in the larger lymphatic vessels. The lymphatic valves, in addition to determining prograde flow as the vessel walls contract, defend more peripheral and thinner walled lymphatics from having to tolerate high intramural pressures. Congenital valvar incompetence (Milroy's disease) induces lymphangiectasia in the unguarded territory and lymphatic dilatation may be patchy.

Thus, if the analogy between Fontan survivors and patients with constrictive pericarditis holds up, liver dysfunction would be mild and manifest only on laboratory testing for most patients. Clinical difficulties arise when the lymphatic system begins to fail, presumably due to progressive valvar incompetence leading to lymphangiectasia and hypoalbuminaemia. The patients who decompensate prematurely may be "worse Fontans" in the sense of running a particularly high venous pressure but they might be unlucky in some other way-some women suffer varicose indignities during their first pregnancy, others not until their fifth. Perhaps intestinal lymphangiectasia relates as much to individual lymphatic intestinal valvar anatomy as to the level of venous hypertension.

\section{Cardiac output, venous hypertension, and low albumin levels}

At a certain level of hypoalbuminaemia cardiovascular physiology begins to fail. The level of venous pressure that can be sustained depends largely on the plasma albumin concentration because albumin is the molecule largely responsible for providing the oncotic pressure that defends circulating plasma volume as blood traverses the capillary spaces. A rule of thumb linking the ceiling of venous pressure with plasma albumin is: albumin in $\mathrm{g} / 100 \mathrm{ml} \times 5.2=$ venous pressure above which pulmonary or systemic oedema is generated. ${ }^{14}$ Thus at a plasma albumin of $2.5 \mathrm{~g} / 100 \mathrm{ml}$, a venous pressure no higher than $13 \mathrm{~mm} \mathrm{Hg}$ can be sustained. Some Fontan patients may need a systemic venous pressure above this to maintain cardiac output and so their cardiac output must fall in the face of hypoalbuminaemia. This ceiling explains why the expected relation between very high systemic venous pressure and very low serum albumin is not observed. ${ }^{26}$

\section{Is the Fontan situation itself a hypercoagulable state?}

Late thromboembolic events have been well documented after the Fontan operation-Rosenthal et al estimated an approximately constant hazard rate of 3.9 events per 100 patient-years. ${ }^{12}$ Virchow proposed three components to thrombogenicity: changes in the vessel wall, changes in blood flow, and changes in the coagulability of the blood. Even in the newly engineered Fontan circuits that aim to minimise stasis in the right heart, abnormal patterns of blood flow are an integral part of the Fontan situation. Recent studies also document a prevalence of protein $\mathrm{C}$ deficiency of over $25 \%$ after four years, a key factor in physiological antithrombotic pathways. ${ }^{2}$ As with the clotting factor abnormalities observed in association with isolated atrial fibrillation, ${ }^{13}$ it is not clear whether the haemodynamic abnormalities are in some way a cause of the blood abnormalities. However, the background in Fontan patients might also be acquired protein $\mathrm{C}$ deficiency as found in chronic liver disease or intestinal protein loss of the small protein $\mathrm{C}$ molecule.

\section{Do Fontan patients require anticoagulation in the} long term?

Kaulitz et al recommend regular screening of clotting factor profiles and "strong anticoagulation" in all Fontan patients with a procoagulant state on paper. ${ }^{2}$ CrommeDijkhuis et al prefer to anticoagulate on clinical grounds if the patient has a low cardiac output or a dilated right atrium. ${ }^{3}$ Trials in atrial fibrillation suggest that a strategy of prescribing warfarin to every patient may not be optimal because of the incidence of bleeding and the significant inconvenience to patients. ${ }^{14}$ Even if formal anticoagulation for all Fontan patients were recommended by physicians anxious to optimise event free survival curves, Fontan adolescents might not see the issues in the same light. Teenagers are not as risk averse as adults; many struggling to maintain their self esteem despite their medical history and current problems, and will resist medication that distances them further from "normality". ${ }^{15}$

\section{The future of the Fontan}

Should the real possibility that circulations operating with high systemic venous pressures gradually and inevitably decompensate as the lymphatic system fails influence attitudes to recommending the Fontan operation to patients who are apparently suitable? These are young people for whom no palliation can offer either the quality of life they yearn for or a realistic chance of a normal life span. More data comparing the exercise capacity of patients palliated with a Fontan-type circuit, with cavo pulmonary and with systemic to pulmonary shunts, would help decision making. However, in communicating the information we must also acknowledge the difference between a middle aged outlook, which values long life, and the inclination of adolescents to attach more value to the quality of the next year of their lives than to long term survival. ${ }^{16}$

KATE BULL

Cardiothoracic Unit,

Great Ormond Street Hospital for Children,

London WC1N 3fH, UK

1 Fontan F, Kirklin KW, Fernandez G, et al. Outcome after a "perfect" Fon$\tan$ operation. Circulation 1990;81:1520-36.

2 Kaulitz R, Luhmer I, Bergmann F, et al. Sequelae after modified Fontan operation: postoperative haemodynamic data and organ function. Heart 1997;78:154-9.

3 Cromme-Dijkhuis AH, Hess J, Hählen K, et al. Specific sequelae after Fontan operation at mid- and long-term follow-up. 7 Thorac Cardiovasc Surg 1993;106:1126-32.

4 Nelson DL, Blaese RM, Strober W, et al. Constrictive pericarditis, intestinal lymphangiectasia, and reversible immunologic deficiency. $\mathcal{F}$ Pediatr 1975;86:548-54

5 Davidson JD, Waldmann TA, Goodman DS, et al. Protein-losing gastroenteropathy in congestive heart failure. Lancet 1961;i:899-902.

6 Strober W, Cohen LS, Waldmann TA, et al. Tricuspid regurgitation. A newly recognized cause of protein-losing enteropathy, lymphocytopenia and immunologic deficiency. Am F Med 1968;44:842-50.

7 Feldt RH, Driscoll DJ, Offord KP, et al. Protein-losing enteropathy after the Fontan operation. $\mathcal{F}$ Thorac Cardiovasc Surg 1996;112:672-80.

8 Kinmonth JB, Sharpey-Schafer EP. Pressure waves in the human thoracic Kinmonth JB, Sharpey-Schafer EP. Pr

9 Benoit JN. Relationships between lymphatic pump flow and total lymph flow in the small intestine. Am ₹ Physiol 1991;261:H1970-78.

10 Drake RE, Abbott RD. Effect of increased neck vein pressure on intestinal lymphatic pressure in awake sheep. Am f Physiol 1992;262:R892-4.

11 Guyton AC, Lindsey AW. Effect of elevated left atrial pressure and decreased plasma protein concentration on the development of pulmonary oedema. Circ Res 1959;7:649.

12 Rosenthal DN, Friedman AH, Kleinman CS, et al. Thromboembolic complications after Fontan operations. Circulation 1995;92(suppl II):287-93.

13 Lip GYH. Does atrial fibrillation confer a hypercoagulable state? Lancet 1995;346:1313-14.

14 Atrial Fibrillation Investigators. Risk factors for stroke and efficacy of antithrombotic therapy in atrial fibrillation. Analysis of pooled data from five randomized controlled trials. Arch Intern Med 194;154:1449-57.

15 Weekes DP. Adolescents growing up chronically ill-a life-span developmental view. Family Comm Health 1995:17:22-34.

16 Bart WM. Adolescent thinking and the quality of life. Adolescence 1983;18: 875-88. 